\title{
A Comprehensive Three-Dimensional Model of the Cochlea *
}

\author{
Edward Givelberg ${ }^{\mathrm{a}}$ \\ a Department of Mechanical Engineering, University of Michigan, Ann Arbor \\ Julian Bunn ${ }^{\mathrm{b}}$ \\ b Center for Advanced Computing Research, California Institute of Technology, \\ Pasadena, USA
}

\begin{abstract}
The human cochlea is a remarkable device, able to discern extremely small amplitude sound pressure waves, and discriminate between very close frequencies. Simulation of the cochlea is computationally challenging due to its complex geometry, intricate construction and small physical size. We have developed, and are continuing to refine, a detailed three-dimensional computational model based on an accurate cochlear geometry obtained from physical measurements. In the model, the immersed boundary method is used to calculate the fluid-structure interactions produced in response to incoming sound waves. The model includes a detailed and realistic description of the various elastic structures present.

In this paper, we describe the computational model and its performance on the latest generation of shared memory servers from Hewlett Packard. Using compiler generated threads and OpenMP directives, we have achieved a high degree of parallelism in the executable, which has made possible several large scale numerical simulation experiments that study the interesting features of the cochlear system. We show several results from these simulations, reproducing some of the basic known characteristics of cochlear mechanics.
\end{abstract}

Key words: Cochlea, Immersed Boundary Method, Navier-Stokes Equations, Parallel Computation, Shared Memory

PACS:

‡ Part of this research was supported by NIH grant RO1-DC04084. 


\section{Introduction}

The cochlea is the part of the inner ear where acoustic signals are transformed into neural pulses which are then signaled to the brain. It is a small organ, the size of a child's marble, which is a miracle of nature's engineering. It is sensitive to signals ranging over more than seven orders of magnitude, from a whisper to an explosion. We can hear sounds ranging in frequency from approximately $10 \mathrm{~Hz}$ to about $20 \mathrm{kHz}$, and distinguish between tones whose frequencies differ by less than a percent. Trained musicians, for example, are capable of differentiating between pure tones of $1000 \mathrm{~Hz}$ and $1001 \mathrm{~Hz}$.

These remarkable signal processing capabilities are achieved by a complicated mechanism involving both interaction of elastic material components immersed in fluid and electro-chemical processes. After decades of research a fascinating picture of the cochlear mechanics has emerged, but the precise nature of the mechanisms responsible for the extreme sensitivity, sharp frequency selectivity and the wide dynamic range of the cochlea remains unknown. The goal of our project is to build computational modeling tools that we hope will assist in the understanding of how the cochlea works. We have constructed a comprehensive three-dimensional computational model of the fluid-structure interactions of the cochlea using the immersed boundary method. This is the first model that incorporates the curved cochlear anatomy based on real physical measurements, uses the non-linear Navier-Stokes equations of viscous fluid dynamics and includes a detailed and realistic description of the various elastic structures present. For example, the helicoidal basilar membrane is modeled as an elastic shell using partial differential equations.

We have developed a suite of software codes to support our studies of the cochlea using the immersed boundary method. These include codes for the generation of simulation input models (implemented in $\mathrm{C}++$ ), the main immersed boundary numerical solver, and those for the analysis and visualization of results (implemented in Java and $\mathrm{C}++$ ). The main workhorse in this suite is the general purpose immersed boundary code, which is written in $\mathrm{C}$ and requires extensive computing resources (CPU power, memory, and available disk space for storage of the results files).

Our present work builds on the first author's implementation of the immersed boundary method for elastic shells [12]. In this paper we outline the construction of the cochlea model and present preliminary results of several large scale numerical experiments. These experiments reproduce some of the basic features of cochlear mechanics and demonstrate the promise of large scale computational modeling approach to answering important questions about nature's miraculous engineering design of the cochlea. 
The rest of the paper is organized as follows. Section 2 is a short introduction to cochlear mechanics, including a brief survey of some of the previous work on cochlear modeling. For a comprehensive up to date introduction to the subject see Geisler's excellent book [11]. Section 3 describes the general methodology of the immersed boundary method. We write down the fluid-structure interaction equations and outline the general first-order numerical method to solve these equations. The method has been extensively tested for different models of the elastic immersed boundary. For more detailed presentations see [31], [30] and [12]. We also discuss briefly the implementation of the immersed boundary algorithm and its optimization. Section 4 describes the construction of the cochlea model. In section 5 we present results of several numerical simulations. Our cochlea model is a work in progress and we conclude with an outline of future directions for this project.

\section{The Cochlea}

\subsection{Cochlear Mechanics}

The cochlea is a small snail-shell-like cavity in the temporal bone, which has two openings, the oval window and the round window. The cavity is filled with fluid and is sealed by two elastic membranes that cover the windows. The spiral canal of the cochlea is divided lengthwise into two passages by the cochlear partition consisting of the bony shelf and the so-called basilar membrane. These passages are the scala vestibuli and scala tympani, and they connect with each other at the apex, called the helicotrema. External sounds set the ear drum in motion, which is conveyed to the inner ear by the ossicles, three small bones of the middle ear, the malleus, incus and stapes. The ossicles function as an impedance matching device, focusing the energy of the ear drum on the oval window of the cochlea. The piston-like motion of the stapes against the oval window displaces the fluid of the cochlea, so generating traveling waves that propagate along the basilar membrane.

Much of what we know about the waves in the cochlea was discovered in the 1940s by Georg von Békésy [45], who carried out experiments in cochleae extracted from human cadavers. Von Békésy studied the cochlea as a passive mechanical filter that utilizes a system of elastic components immersed in a fluid for analysis of incoming sounds. He observed that a pure tone input generates a traveling wave propagating along the basilar membrane. The wave amplitude rises gradually, reaching a peak at a specific location along the membrane, after which it decays rapidly. The peak location depends on the frequency of the input tone, with high frequencies peaking close to the stapes, and the lower frequencies further towards the apex. This "place principle" 
is a crucial mechanism of frequency analysis in the cochlea. Resting on the basilar membrane is the microscopic organ of Corti, a complicated structure containing sensory receptors called hair cells. The hair cells detect fluid motion and provide input to the afferent nerve fibers that send action potentials to the brain. Thus, a pure tone input activates only a specific group of hair cells depending on its frequency, with the characteristic frequency locations monotonically decreasing along the basilar membrane from the stapes to the helicotrema.

Von Békésy found that the basilar membrane's elastic properties play an important role in the wave mechanics of the cochlea. Despite its name, the basilar membrane is in fact an elastic shell whose compliance per unit length increases exponentially with length. (Unlike an elastic membrane, an elastic shell is not under inner tension, i.e. when it is cut the edges do not pull apart. The compliance of an elastic shell is defined as the amount of volume displaced per unit pressure difference across the surface of the shell.) Von Békésy's experiments further revealed that the wave propagates along the basilar membrane in the direction of increasing compliance regardless of the location of the source of excitation in the fluid. This phenomenon came to be known as "the traveling wave paradox" and it is very important because a significant part of our hearing depends on bone conduction, where the input to the cochlea is coming not through the stapes, but through the vibration of the bony walls. Bone conduction is easily demonstrated by placing a vibrating tuning fork in contact with the forehead, resulting in the subject hearing the tone of the fork frequency.

Mathematically, the macro-mechanical system of the cochlea can be described by the Navier-Stokes equations of incompressible fluid mechanics coupled with equations modeling the elastic properties of the basilar membrane and the membranes of the oval and the round windows. The mathematical problem of solving this system of partial differential equations on a three-dimensional domain with intricate curved geometry is very difficult. Since the displacements of the basilar membrane are extremely small (on a nanometer scale), the system operates in a linear regime. Analysis shows that the waves in the cochlea resemble shallow water waves [24].

While the macro-mechanics of the cochlea break up the incoming sound into its frequency components, it was suggested as early as 1948 that a passive mechanical system alone cannot explain the extreme sensitivity and frequency selectivity of the cochlea; some kind of amplification is necessary [13]. Indeed, analysis of cochlear macro-mechanics indicates that the traveling wave focusing is not sufficiently sharp, with some estimates suggesting that, at its threshold, human hearing is about a hundred times more sensitive than what would be expected from a passive macro-mechanical filter of the cochlea.

In 1967 Johnstone and Boyle [16] utilized the Mössbauer effect to carry out 
measurements of the basilar membrane vibrations in vivo, with more than 100-fold improvement in resolution over von Békésy's measurements. These measurements revealed that in the live cochlea the peak of the wave envelope is, in fact, well localized [36]. Since then the fine tuning of the cochlea has been confirmed experimentally in many studies, e.g. $[37,39,18]$. This led to attempts to understand the mechanical and electro-chemical processes at the level of the hair cells and to a renewed interest in the conjecture that the cochlea contains an energy source and acts as a mechanical amplifier.

Further indirect support for the active amplification hypothesis was found with the discovery of the existence of otoacoustic emissions $[20,17]$. Otoacoustic emissions may be recorded during or after acoustical stimulation using a sensitive microphone placed close to the ear drum. Emissions can also be detected when electric current is applied to the cochlea [27]. Spontaneous otoacoustic emissions (SOAEs) occur in humans and other species. In the severe cases SOAEs are the cause of objective tinnitus, a common complaint of patients with "ringing ears". Experiments show that a SOAE can be suppressed when a stimulus tone is presented at a nearby frequency. An isosuppression tuning curve is a curve obtained by measuring the otoacoustic emission while varying the amplitude and the frequency of the stimulus. In both mammalian and nonmammalian cochleae SOAEs and the process responsible for sharp frequency selectivity display similar characteristics. Measurements show that the isosuppression tuning curve closely resembles an ordinary tuning curve [8], which measures responsiveness to acoustical stimulus, leading to the conjecture that the cochlear amplifier is also the source of the SOAEs.

Presently, understanding the nature of the active mechanism in the cochlea is the main open problem of hearing research. The live cochlea is a remarkable highly non-linear filter, but its function crucially depends on the underlying linear filter of the passive cochlear mechanics, which is still not sufficiently well understood. Basic questions about the role of geometry and the elastic properties of the basilar membrane in cochlear mechanics remain open. Answering such questions is important not only to our understanding of the cochlea, but also in solving important engineering problems, such as a cochlear transducer design (see [34]).

\subsection{Cochlear Models}

Extensive research in cochlear modeling has been carried out over the years. Because of mathematical difficulty mostly simplified one or two-dimensional models that sought to incorporate some aspects of cochlear mechanics have been proposed. 
Early one-dimensional "transmission line" models of the cochlea [48], [33], [10] have assumed that the fluid pressure is constant over a cross section of the cochlear channel. The fluid is assumed to be incompressible and inviscid, and the basilar membrane is modelled as a damped, forced harmonic oscillator with no elastic coupling along its length. Qualitatively, this model has been shown to capture the basic features of the basilar membrane response. Quantitatively, however, it yields large discrepancies with measurement results [46].

Two-dimensional models by Ranke [35] and Zwislocki [47] make similar assumptions on the cochlear fluid and the basilar membrane. Ranke's model uses a deep water approximation, while Zwislocki used the shallow water theory in his model. These models were further developed in [40], [23], [4], [3] and other works. The most rigorous analysis of a two-dimensional model with fluid viscosity was carried out by Leveque, Peskin and Lax in [24]. Their cochlea is represented by a plane (i.e. a strip of infinite length and infinite depth) and the basilar membrane, by an infinite line dividing the plane into two halves. The linearized equations are reduced to a functional equation by applying the Fourier transform in the direction parallel to the basilar membrane and then solving the resulting ordinary differential equations in the normal direction. The functional equation derived in this way is solved analytically, and the solution is evaluated both numerically and also asymptotically (by the method of stationary phase). This analysis reveals that the waves in the cochlea resemble shallow water waves, i.e. ripples on the surface of a pond. A distinctive feature of this paper is the (then speculative) consideration of negative basilar membrane friction, i.e., of an amplification mechanism operating within the cochlea.

Other two-dimensional models incorporate more sophisticated representations of the basilar membrane, using, for example, elastic beam and plate theory ([6], [38], [19], [2], [41], [15], [7], [14]). Three-dimensional models were considered by Steele and Taber [42] and de Boer [9], who used asymptotic methods and computations, obtaining an improved fit of the experimental data. Their experience seems to indicate that geometry may play a significant role in the problem. In particular, the effect of the spiral coiling of the cochlea on the wave dynamics remains unresolved. It has been considered by several authors (see [44], [25], [43], [26]).

With the development of more powerful computers it became possible to construct more detailed computational models of the cochlea. A two-dimensional computational model of the cochlea was constructed by Beyer [5]. In this model the cochlea is a flat rectangular strip divided into two equal halves by a line which represents the basilar membrane. The fluid is modelled by the full Navier-Stokes equations with viscosity terms, but elastic coupling along the basilar membrane is not incorporated. Beyer has used a modification of Peskin's immersed boundary method, originally developed for modeling 
the fluid dynamics of the heart [29]. Several three-dimensional computational models have been reported, such as Kolston's model [21,22], intended to simulate the micro-mechanics of the cochlear partition in the linear regime (i.e. near the threshold of hearing), and Parthasarati, Grosh and Nuttal's [28] hybrid analytical-computational model using WKB approximations and finiteelement methods.

\section{The Immersed Boundary Method}

The primary method used in our construction of the cochlea model is the immersed boundary method of Peskin and McQueen. It is a general numerical method for modeling an elastic material immersed in a viscous incompressible fluid. It has proved to be particularly useful for computer simulation of various biofluid dynamic systems. In this section we outline the general framework of the immersed boundary method. So far most applications of the method have treated the elastic (and possibly active) biological tissue as a collection of elastic fibers immersed in a viscous incompressible fluid. For details of this formulation of the method together with references to many applications see [31] and [30]. The immersed boundary framework is suitable for modeling not only elastic fibers, but also different elastic materials having complicated structure. The cochlea model makes an essential use of the immersed boundary method for shells, as developed in [12]. The main advantage of the immersed boundary method is its conceptual simplicity: the viscous incompressible fluid is described by the Navier-Stokes equations, the geometry of the model mirrors the real-life curved three-dimensional cochlear geometry and models for elastic and active material components can be naturally integrated.

\subsection{The Equations of the Model}

The immersed boundary method is based on a Lagrangean formulation of the fluid-immersed material system. The fluid is described in the standard cartesian coordinates on $\mathbf{R}^{3}$, while the immersed material is described in a different curvilinear coordinate system. Let $\rho$ and $\mu$ denote the density and the viscosity of the fluid, and let $\mathbf{u}(\mathbf{x}, t)$ and $p(\mathbf{x}, t)$ denote its velocity and pressure, respectively. The Navier-Stokes equations of a viscous incompressible fluid are:

$$
\begin{aligned}
\rho\left(\frac{\partial \mathbf{u}}{\partial t}+\mathbf{u} \cdot \nabla \mathbf{u}\right) & =-\nabla p+\mu \nabla^{2} \mathbf{u}+\mathbf{F} \\
\nabla \cdot \mathbf{u} & =0
\end{aligned}
$$


where $\mathbf{F}$ denotes the density of the body force acting on the fluid. For example, if the immersed material is modeled as a thin shell, then $\mathbf{F}$ is a singular vector field, which is zero everywhere, except possibly on the surface representing the shell. The numerical method uses a discretization of the Navier-Stokes equations (1) and (2) on a periodic rectangular grid.

Let $\mathbf{X}(\mathbf{q}, t)$ denote the position of the immersed material in $\mathbf{R}^{3}$. For a shell, $\mathbf{q}$ takes values in a domain $\Omega \subset \mathbf{R}^{2}$, and $\mathbf{X}(\mathbf{q}, t)$ is a 1-parameter family of surfaces indexed by $t$, i.e., $\mathbf{X}(\mathbf{q}, t)$ is the middle surface of the shell at time $t$. Let $\mathbf{f}(\mathbf{q}, t)$ denote the force density that the immersed material applies on the fluid. Then

$$
\mathbf{F}(\mathbf{x}, t)=\int \mathbf{f}(\mathbf{q}, t) \delta(\mathbf{x}-\mathbf{X}(\mathbf{q}, t)) d \mathbf{q}
$$

where $\delta$ is the Dirac delta function on $\mathbf{R}^{3}$. This equation merely says that the fluid feels the force that the immersed material exerts on it, but it is important in the numerical method, where it is one of the equations determining fluidmaterial interaction. The other interaction equation is the no-slip condition for a viscous fluid:

$$
\begin{aligned}
\frac{\partial \mathbf{X}}{\partial t} & =\mathbf{u}(\mathbf{X}(\mathbf{q}, t), t) \\
& =\int \mathbf{u}(\mathbf{x}, t) \delta(\mathbf{x}-\mathbf{X}(\mathbf{q}, t)) d \mathbf{x}
\end{aligned}
$$

The system has to be completed by specifying the force $\mathbf{f}(\mathbf{q}, t)$ of the immersed material. In a complicated system such as the cochlea the immersed material consists of many different components: membranes, bony walls, an elastic shell representing the basilar membrane, and various cells of the organ of Corti, including outer hair cells, which may actively generate forces. For each such component it is necessary to specify its own computation grid and an algorithm to compute its force $\mathbf{f}$. It is in the specification of these forces that models for various system components integrate into the macro-mechanical model.

\subsection{The Numerical Method}

We describe here a first-order immersed boundary numerical scheme, which is the easiest to implement and has the important advantage of modularity: incorporating various models of immersed elastic material is straightforward.

Let $\Delta t$ denote the duration of a time step. It will be convenient to denote the time step by the superscript. For example $\mathbf{u}^{n}(\mathbf{x})=\mathbf{u}(\mathbf{x}, n \Delta t)$. At the beginning 
of the $n$-th time step $\mathbf{X}^{n}$ and $\mathbf{u}^{n}$ are known. Each time step proceeds as follows.

(1) Compute the force $\mathbf{f}^{n}$ that the immersed boundary applies to the fluid. For simple materials, such as fibers, this is a straightforward computation (see [31]). For a detailed description of a shell immersed boundary force computation see [12].

(2) Use (3) to compute the external force on the fluid $\mathbf{F}^{n}$.

(3) Compute the new fluid velocity $\mathbf{u}^{n+1}$ from the Navier Stokes equations.

(4) Use (4) to compute the new position $\mathbf{X}^{n+1}$ of the immersed material.

We shall now describe in detail the computations in steps $2-4$, beginning with the Navier-Stokes equations.

The fluid equations are discretized on a rectangular lattice of mesh width $h$. We will make use of the following difference operators which act on functions defined on this lattice:

$$
\begin{aligned}
D_{i}^{+} \phi(\mathbf{x}) & =\frac{\phi\left(\mathbf{x}+h \mathbf{e}_{i}\right)-\phi(\mathbf{x})}{h} \\
D_{i}^{-} \phi(\mathbf{x}) & =\frac{\phi(\mathbf{x})-\phi\left(\mathbf{x}-h \mathbf{e}_{i}\right)}{h} \\
D_{i}^{0} \phi(\mathbf{x}) & =\frac{\phi\left(\mathbf{x}+h \mathbf{e}_{i}\right)-\phi\left(\mathbf{x}-h \mathbf{e}_{i}\right)}{2 h} \\
\mathbf{D}^{\mathbf{0}} & =\left(D_{1}^{0}, D_{2}^{0}, D_{3}^{0}\right)
\end{aligned}
$$

where $i=1,2,3$, and $\mathbf{e}_{1}, \mathbf{e}_{2}, \mathbf{e}_{3}$ form an orthonormal basis of $\mathbf{R}^{3}$.

In step 3 we use the already known $\mathbf{u}^{n}$ and $\mathbf{F}^{n}$ to compute $\mathbf{u}^{n+1}$ and $p^{n+1}$ by solving the following linear system of equations:

$$
\begin{aligned}
\rho\left(\frac{\mathbf{u}^{n+1}-\mathbf{u}^{n}}{\Delta t}+\sum_{k=1}^{3} u_{k}^{n} D_{k}^{ \pm} \mathbf{u}^{n}\right) & =-\mathbf{D}^{0} p^{n+1}+\mu \sum_{k=1}^{3} D_{k}^{+} D_{k}^{-} \mathbf{u}^{n+1}+\mathbf{F}^{n} \\
\mathbf{D}^{0} \cdot \mathbf{u}^{n+1} & =0
\end{aligned}
$$

Here $u_{k}^{n} D_{k}^{ \pm}$stands for upwind differencing:

$$
u_{k}^{n} D_{k}^{ \pm}= \begin{cases}u_{k}^{n} D_{k}^{-}, & u_{k}^{n}>0 \\ u_{k}^{n} D_{k}^{+}, & u_{k}^{n}<0\end{cases}
$$

Equations (9) and (10) are linear constant coefficient difference equations and, therefore, can be solved efficiently with the use of the Fast Fourier Transform algorithm.

We now turn to the discretization of equations (3), (4). Let us assume, for 
simplicity, that $\Omega \subset \mathbf{R}^{2}$ is a rectangular domain over which all of the quantities related to the shell are defined. We will assume that this domain is discretized with mesh widths $\Delta q_{1}, \Delta q_{2}$ and the computational lattice for $\Omega$ is the set

$$
\mathbf{Q}=\left\{\left(i_{1} \Delta q_{1}, i_{2} \Delta q_{2}\right) \mid i_{1}=1 \ldots n_{1}, \quad i_{2}=1 \ldots n_{2}\right\}
$$

In step 2 the force $\mathbf{F}^{n}$ is computed using the following equation.

$$
\mathbf{F}^{n}(\mathbf{x})=\sum_{\mathbf{q} \in \mathbf{Q}} \mathbf{f}^{n}(\mathbf{q}) \delta_{h}\left(\mathbf{x}-\mathbf{X}^{n}(\mathbf{q})\right) \Delta \mathbf{q}
$$

where $\Delta \mathbf{q}=\Delta q_{1} \Delta q_{2}$ and $\delta_{h}$ is a smoothed approximation to the Dirac delta function on $\mathbf{R}^{3}$ described below.

Similarly, in step 4 updating the position of the immersed material $\mathbf{X}^{n+1}$ is done using the equation

$$
\mathbf{X}^{n+1}(\mathbf{q})=\mathbf{X}^{n}(\mathbf{q})+\Delta t \sum_{\mathbf{x}} \mathbf{u}^{n+1}(\mathbf{x}) \delta_{h}\left(\mathbf{x}-\mathbf{X}^{n}(\mathbf{q})\right) h^{3}
$$

where the summation is over the lattice $\mathbf{x}=(h i, h j, h k)$, where $i, j$ and $k$ are integers.

The function $\delta_{h}$ which is used in (11) and (12), is defined as follows:

$$
\delta_{h}(\mathbf{x})=h^{-3} \phi\left(\frac{x_{1}}{h}\right) \phi\left(\frac{x_{2}}{h}\right) \phi\left(\frac{x_{3}}{h}\right)
$$

where

$$
\phi(r)=\left\{\begin{array}{cl}
\frac{1}{8}\left(3-2|r|+\sqrt{1+4|r|-4 r^{2}}\right) & |r| \leq 1 \\
\frac{1}{2}-\phi(2-|r|) & 1 \leq|r| \leq 2 \\
0 & 2 \leq|r|
\end{array}\right.
$$

For an explanation of the construction of $\delta_{h}$ see [31].

\subsection{Implementation and Optimization of Immersed Boundary Computations}

Our main simulation code implements the first-order immersed boundary algorithm described above. It is written in $\mathrm{C}$ and has been optimized to run on several platforms: the Silicon Graphics Origin 2000 parallel architecture, the Cray T90 vector-parallel machine and the HP V-class and Superdome systems. There is also a version for a PC running Windows, suitable for testing small models. The simulation code takes as input a set of files which contain the 
description of the geometry and the material properties of the system. These files are created before the start of the simulation by the model generation software. Once the model has been read, the simulation enters a computation loop over the required number of time steps, generating output files containing information about the state of the simulated model.

The complexity of an immersed boundary computation is determined by the sizes of the fluid and immersed boundary grids and by the size of the time step. To prevent fluid leakage the mesh width of the material grids is taken to equal approximately half the fluid grid mesh width. (For more on volume conservation in immersed boundary calculations see [32]). The computation of the material forces is relatively inexpensive in time. The bulk of the computation is related to the fluid equations and to the fluid-immersed material interaction. The solution of the discretized fluid equations (9), (10) uses the Fast Fourier Transform algorithm. The other two demanding parts of each time step required development of a specialized algorithm that carefully partitions the fluid and the material grids into portions that are distributed amongst the available processors. This is done in such a way as to ensure that no two processors operate simultaneously on the same portion of the data. Numerical consistency conditions require reducing the time step when the space mesh width is decreased. Our empirical convergence study indicated that the computation is numerically stable when the time step is proportional to the mesh width. For example, decreasing the mesh width by a factor of 2 necessitates decreasing the time step by approximately a factor of 2 as well. Thus rescaling a model with a $128^{3}$-point fluid grid to a $256^{3}$-point model requires approximately 8 times as much memory and 16 times as much CPU time [12].

For large scale simulations, such as that of the full cochlea model, extensive optimization of the immersed boundary code was necessary so as to reduce the elapsed time to a length that allowed useful experiments to be completed in a manageble time. The code is instrumented with calls to system timing routines, this information proving invaluable during porting and tuning of the software on different architectures. The largest and most recent immersed boundary numerical experiments have been carried out on the 32 processor HP Superdome installed at the Center for Advanced Computing Research (CACR) at Caltech. The HP 9000 Superdome at CACR contains 32 RISC processors arranged in a cell-based hierarchical crossbar architecture, with each cell consisting of 4 cpus with $8 \mathrm{~Gb}$ of memory and an I/O sub-system. This architecture supports the shared memory programming model and the code efficiency was achieved primarily through the use of OpenMP parallelization directives. We used several software tools such as the HP CXperf and the KAI Guideview to examine the parallel efficiency of every section in the code and to identify data cache and TLB misses. Figure 1 shows the excellent scaling behavior of the simulation as a function of the number of allocated processors. Following this work, the "wall-clock" time per step of the simulation iof a 
$256 \times 256 \times 128$-based cochlea model is approximately 1.3 seconds when running on all 32 processors of the HP Superdome.

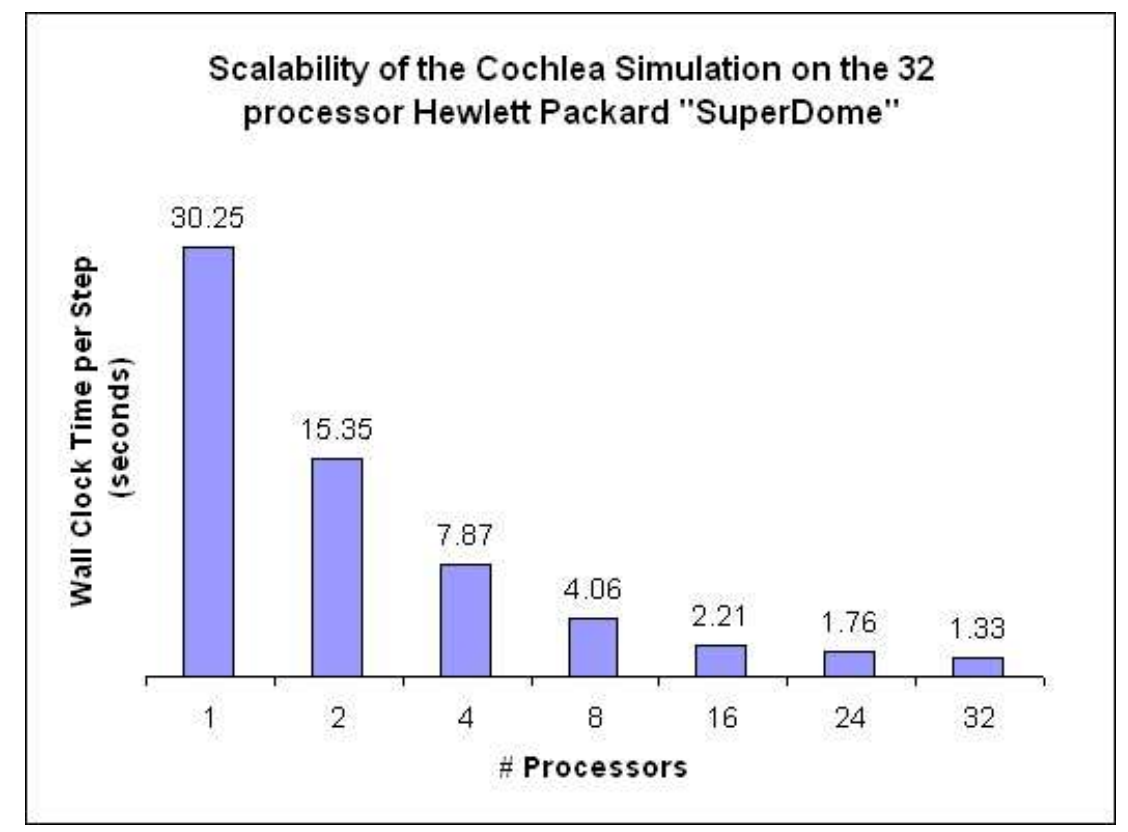

Fig. 1. Execution wall-clock time per time step vs. number of processors.

\section{The Cochlea Model}

The size of the human cochlea is about $1 \mathrm{~cm} \times 1 \mathrm{~cm} \times 1 \mathrm{~cm}$, while the human basilar membrane is approximately $3.5 \mathrm{~cm}$ long, is very thin and very narrow: its width grows from about $150 \mu \mathrm{m}$ near the stapes to approximately $560 \mu \mathrm{m}$ near the helicotrema. Since the mesh width of the basilar membrane should approximately equal half the mesh width of the fluid grid (see section 3), a fluid grid of at least $256^{3}$ points is necessary to adequately resolve the width of the basilar membrane. The geometric model of the cochlear anatomy is based on measurements that include the position of the center line of the basilar membrane, its width and the cross-sectional area of the scalae. There are six surfaces in this model: the basilar membrane, the spiral bony shelf, the tubular walls of the scala vestibuli and the scala tympani and the semi- 
elliptical walls sealing the cochlear canal and containing the oval and the round window membranes (see Figure 2). The basilar membrane is modeled



Fig. 2. A rendering of the geometric model of the cochlea with several parts of the outer shell removed in order to expose the cochlear partition consisting of the narrow basilar membrane and the bony shelf. The round window is located directly below the oval window and in this picture it is partially obscured by the cochlear partition.

as an elastic shell following the prototype tested earlier (see [12]). It is $3.5 \mathrm{~cm}$ long and we assume its width grows linearly from $0.0015 \mathrm{~cm}$ at the stapes to $0.0052 \mathrm{~cm}$ at the helicotrema. The elastic force that the membrane exerts on the surrounding fluid is given by a fourth order partial differential operator acting on the vector field that measures the displacement of the membrane from its elastic equilibrium. We have assumed that the Lame coefficients of the shell are constant and the variable compliance of the basilar membrane is due to its changing width and thickness. We have used this shell equation to estimate the thickness necessary to achieve the increasing compliance measured by von Békésy. Indeed with only a slight variation in the thickness the estimated compliance per unit length increases as $e^{\lambda x}$, where $\lambda^{-1}=0.7 \mathrm{~cm}$. The oval window and the round window membranes are also modeled as elastic shells, but unlike in the case of the basilar membrane, the compliance of each of these shells is constant throughout the shell. The windows are geometrically identical: each is modeled by a rectangular grid, all of whose points outside a given circle are fixed. Hence each such grid represents a circular plate within a rectangular piece of a bone. 
The model building programs generate the approximate cochlear geometry, the material properties of various surfaces and the set of parameters describing the desired simulation tests. The six surfaces of immersed material in the cochlea model are partitioned into 31 computational grids comprising approximately 750,000 points in total. In addition to the basilar membrane and the windows' grids described above there are 28 grids modeling the bony surfaces. The partitioning of the bony surfaces into 28 rectangular grids was necessary to minimize the total number of material points while maintaining an approximately uniform distance between these points.

The passive cochlea is essentially a linear mechanical filter (see section 2) and the displacements occuring within the cochlea are too small to be seen without magnification. In our numerical experiments the displacements of the basilar membrane are measured on a nano-meter scale. The immersed boundary method is particularly suitable for such a simulation since it possesses a subgrid resolution (the material grid mesh width of our cochlea model is about $20 \mu \mathrm{m})$. Our numerical algorithm however uses the fully non-linear system of Navier-Stokes equations rather than the linearized system because the computational cost of solving the linearized equations in the immersed boundary method would be essentially the same as the cost of solving the fully non-linear system.

The codes which are used to analyse and visualise the cochlear geometry and the simulated dynamics include a $\mathrm{C}++$ /OpenGL tool that runs on SGI and a Java/Java3D tool that runs on most platforms. These tools take as input the vertex coordinates for all computational grids in the cochlea model from the result files for each time step in the simulation. The tools generate a full $3 \mathrm{D}$ rendering of the model geometry. Since the displacements occuring within the cochlea are very small they are color-coded to reveal the dynamics of the system. Our graphics tools also enable us to magnify the displacements to make them visible on the screen. An essential insight into the basilar membrane dynamics is provided by the plot of the normal displacement of its center line (see Figures 4, 5). Other Java tools display various important characteristics of the system dynamics, such as the response of individual points on the basilar membrane as a function of time. All of the graphics tools have built-in facilities for generating animation.

The construction of the full cochlea model has been undertaken in stages with the individual components tested separately prior to the model assembly. The basilar membrane model is described in detail in [12]. The oval window functions as the input window of the cochlea. We simulate the pressure of the stapes against it by prescribing an external force vector field on the window grid. This force is orthogonal to the surface of the window and for a pure tone input its magnitude varies sinusoidally. No force is prescribed on the round window. 
Since many of the numerical experiments with the full cochlea model require days of computing we have made a small modification of the cochlear anatomy in our model. In the altered model the cochlea is packed more tightly and the whole structure fits in a half-cube of size $1 \mathrm{~cm} \times 1 \mathrm{~cm} \times 0.5 \mathrm{~cm}$ rather than a $1 \mathrm{~cm}^{3}$ cube, making it possible to use a fluid grid of $256 \times 256 \times 128$ points. This configuration requires about 1.5 Gigabytes of main memory and the total time needed to simulate a single time step is reduced approximately in half. The main difference between the "half-cube model" and our original model is that in the former model the basilar membrane if flat. I.e. its spiraling shape is completely contained within a plane. All of our test simulations reported in the next section have been carried out with the half-cube model.

\section{$5 \quad$ Numerical Experiments}

The good efficiency of the immersed boundary solver allowed us to complete several large numerical experiments. In this section we present preliminary results from four such experiments. We are continuing this work and a more detailed exposition of the collected data and its analysis will be published in our next paper.

Each of the experiments presented here consisted of applying a pure tone input of a given frequency at the stapes and studying the subsequent motion of the basilar membrane. The input at the stapes was simulated by specifying an external periodic force vector field on the oval window grid. A very small time step of 30 nano-seconds was chosen to guarantee numerical stability and good detail. The choice of the time step was made as a result of the convergence study of the system carried out in [12]. Our initial aim in the numerical experiments was to reproduce the qualitative characteristics of cochlear mechanics predicted by asymptotic analysis and previously reported computational models. Consequently, we have attempted to capture the steady state response of the basilar membrane to pure tones and in each of our experiments we have run the system for the duration equivalent to several input cycles. For example, for a $10 \mathrm{kHz}$ input tone we have run the system for more than 30,000 time steps, equivalent to $0.9 \mathrm{msec}$ of total simulated time. On a 16 processor SuperDome this computation required more than 20 hours to complete. Every 10th time step of the simulation the program generated an output file containing the instantaneous position of the computation grids. The total amount of storage required is measured in tens of Gigabytes of disk space. Correspond-

ingly, the $2 \mathrm{kHz}$ experiment required five times as much computational time and storage.

We have verified that all of our experiments have been carried in the system's linear regime, i.e. the input force at the stapes was kept very small, resulting 


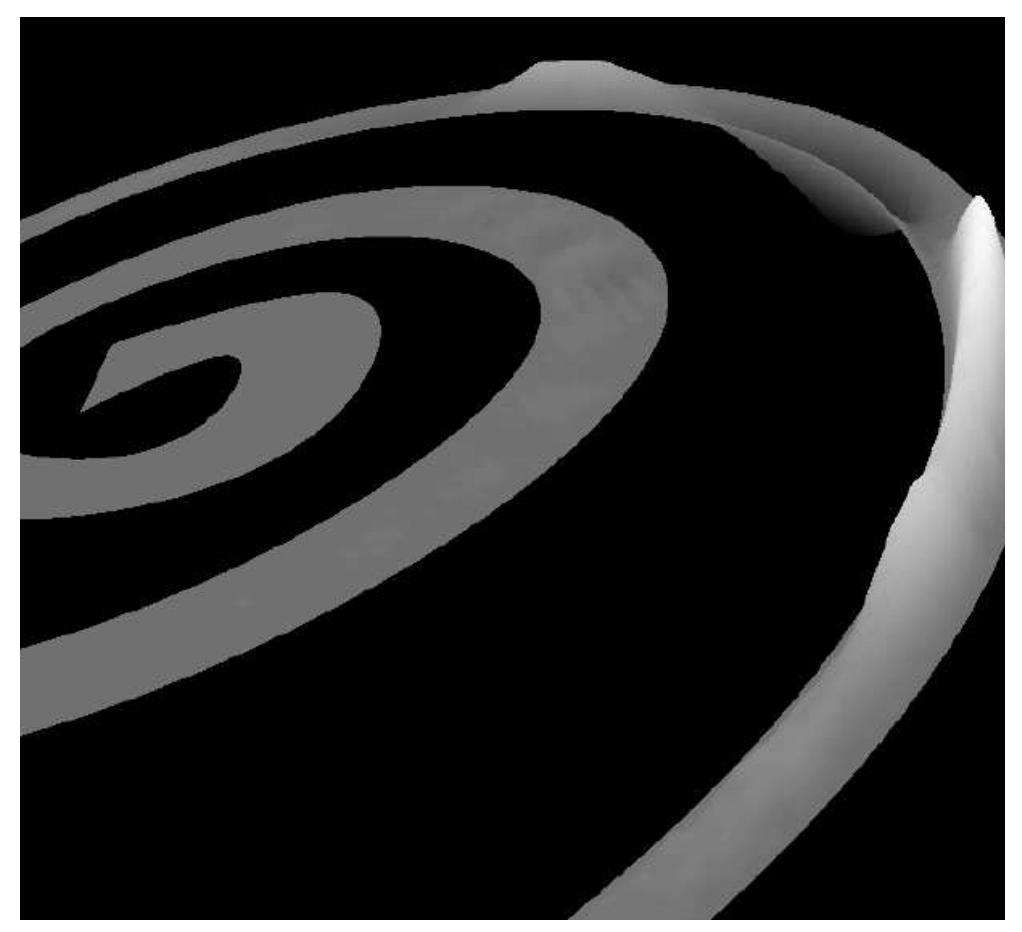

Fig. 3. A close-up snapshot of the traveling wave propagating along the basilar membrane. The magnitude of the basilar membrane displacement has been amplified in the normal direction.

in the basilar membrane displacements on the order of nano-meters. Indeed, increasing the force by a factor of 10 resulted in basilar membrane displacements almost exactly ten times bigger. Since in each experiment the system was started from rest, we have observed initial oscillatory transient response, which was followed by the smoothing of the traveling wave. A close-up snapshot of the traveling wave propagating along the basilar membrane is shown in Figure 3. The wave magnitude has been amplified in the direction of the normal to the basilar membrane.

Much information about the traveling wave is revealed in the plot of the centerline of the basilar membrane. Four such plots are shown in Figure 4 and Figure 5. Figure 4 shows the results of the experiments with input frequency of $15 \mathrm{kHz}$ (top plot) and $10 \mathrm{kHz}$ (bottom plot), and Figure 5 shows the results of the $5 \mathrm{kHz}$ and the $2 \mathrm{kHz}$ experiments. In each plot an instantaneous position of the centerline of the basilar membrane is shown, as well as the wave envelope computed over a range of time steps.

Our experiments reproduced the following characteristic features of cochlear mechanics: In each instance, in response to a pure tone input frequency, we 

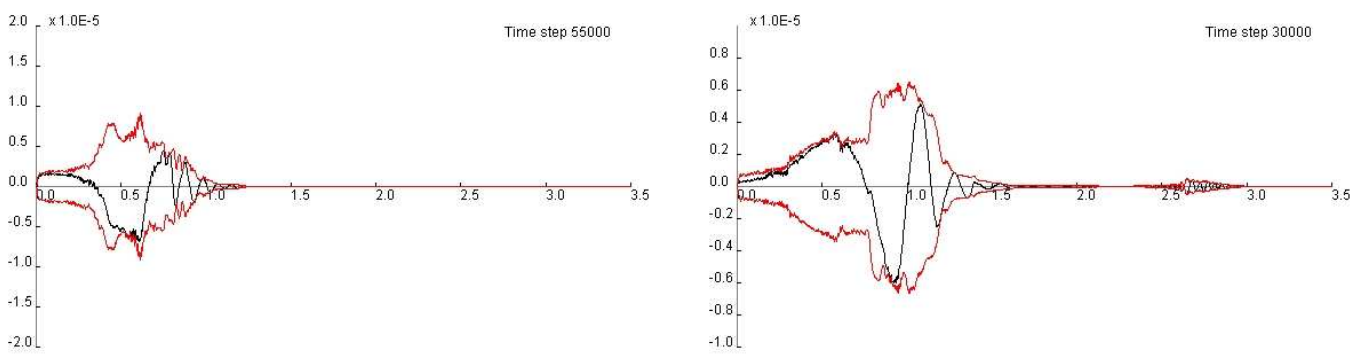

Fig. 4. A snapshot of the center line of the basilar membrane response and wave envelope. The top plot shows the response to the $15 \mathrm{kHz}$ input sound. The wave snapshot is taken after 55000 time steps $(1.65 \mathrm{msec})$. The wave envelope was computed over time steps 45,000 - 55,000. The bottom plot shows a $10 \mathrm{kHz}$ experiment. The wave is shown after 30,000 time steps and the envelope was computed over time steps $20,000-30,000$. The unit of vertical scale is $10^{-5} \mathrm{~cm}$.
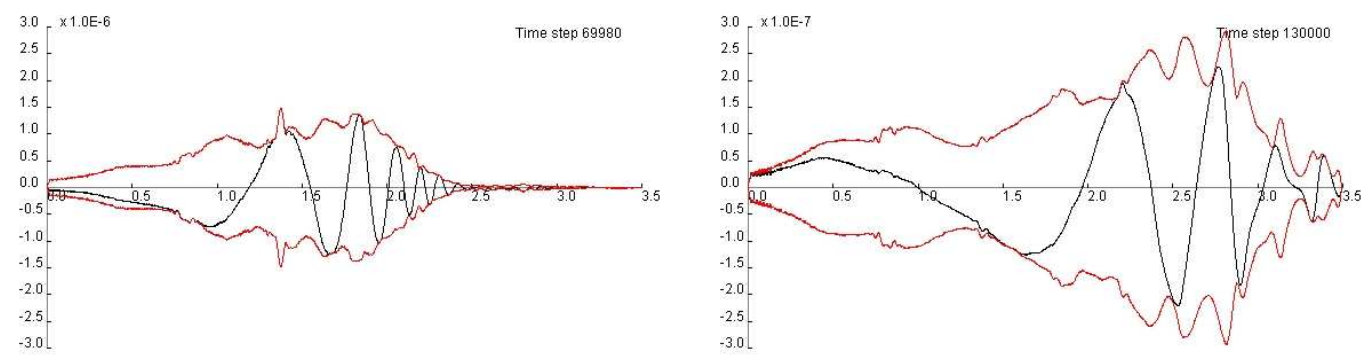

Fig. 5. A snapshot of the center line of the basilar membrane response and wave envelope. The top plot shows the response to the $5 \mathrm{kHz}$ input sound. The wave snapshot is taken after 70000 time steps. The wave envelope was computed over time steps $60,000-70,000$. The bottom plot shows a $2 \mathrm{kHz}$ experiment. The wave is shown after 100,000 time steps and the envelope was computed over time steps $100,000-130,000$. The unit of vertical scale is $10^{-6} \mathrm{~cm}$ and $10^{-7} \mathrm{~cm}$ in the top and in the bottom plots, respectively.

have observed a traveling wave propagating from the stapes in the direction of the helicotrema. The amplitude of the wave is gradually increasing until it reaches a peak at a characteristic location along the basilar membrane de- 
pending on the input frequency. The speed of the wave is sharply reduced as it propagates further along the basilar membrane. The higher the input frequency, the closer the peak of the wave is to the stapes. Furthermore, after reaching the peak the wave drops off sharply, essentially shutting down. Since no active mechanism has been incorporated into the present model yet, the observed traveling wave is not sharply focused.

It is interesting to note that while the computed traveling wave is smooth, its envelope is generally not smooth. The $10 \mathrm{kHz}$ experiment shown in Figure 4 is a particularly striking example, with the envelope turning $90^{\circ}$ sharply in the vertical direction approximately at the $0.75 \mathrm{~cm}$ location along the basilar membrane. We believe that further testing is necessary and more data must be collected from numerical experiments before further conclusions about the shape of the traveling wave envelope can be drawn.

The interested reader is invited to view several animations of our results by visiting our web site[1].

\section{Summary and Conclusions}

We have constructed a comprehensive three-dimensional computational model of the passive cochlea using the immersed boundary method. Extensive optimization and parallelization made it possible to complete several large scale numerical simulations on a 32-processor shared memory HP Superdome computer. Together with the previous demonstration of the traveling wave paradox (see [12]), the pure tone experiments reported in this paper capture the most important properties of the cochlear macro-mechanics.

We would like to note that our results are of somewhat preliminary nature. We are continuing to test the model and plan to complete more numerical experiments in order to compare our simulation results with the available experimental and modeling data. We believe our results demonstrate the promise of large scale computational modeling approach to the study of cochlear mechanics.

The cochlea is a very small and delicate organ and it is very difficult to study experimentally. Many of the important questions of cochlear mechanics are mathematically very complicated, but they can be studied using numerical simulation. Two such general questions concern the effects of the geometry and of the elastic properties of various cochlear components on the dynamics. It is easy to test cochlear models with different geometries such as a straightened out model. Testing different elastic models for the cochlear components is somewhat more involved since it generally requires testing each such model 
separately within the immersed boundary framework. It is nevertheless a feasible project, which is straightforward for the expert.

Going beyound the questions of passive cochlear macro-mechanics we would like to incorporate an active mechanism into our model. The function of the live cochlea undoubtedly depends on the combination of passive cochlear mechanics with an active mechanism. A comprehensive model of the passive cochlea is therefore a necessary first step towards modeling the active live cochlea. It is interesting to note that refining the mesh width of the computational grids by a factor of 2 yields a material mesh width of approximately $10 \mu \mathrm{m}$. This mesh width is small enough to allow much of the structure of the organ of Corti to be incorporated into the model. Such a refined model is not yet feasible since it would require approximately 16 times more computing power than our present model. Indeed, the large scale of computations is presently the biggest obstacle to progress in cochlear modeling, but this is, no doubt, a temporary obstacle. Continuing progress in hardware and software will make a construction of even the refined cochlear model possible soon.

\section{Acknowledgements}

The first author would like to thank Charlie Peskin for suggesting the project and for his guidance through its initial phase, and Karl Grosh for many helpful discussions. We would like to thank Mahesh Rajan for his help with optimizing the numerical solver on the HP machines, and Sarah Emery Bunn for her assistance in the preparation of the manuscript.

\section{References}

[1] http://pcbunn.cacr.caltech.edu/cochlea/.

[2] P. Allaire, S. Raynor, and M. Billone. J. Acoust. Soc. Am., 55:1252-1258, 1974.

[3] J. B. Allen. Two-dimensional cochlear fluid model: New results. J. Acoust. Soc. Am., 61:110-119, 1977.

[4] J. B. Allen and M. M. Sondhi. Cochlear macromechanics: time domain solutions. J. Acoust. Soc. Am., 66:123-132, 1979.

[5] R. P. Beyer. A computational model of the cochlea using the immersed boundary method. J. Comp. Phys., 98:145-162, 1992. 
[6] B. P. Bogert. Determination of the effects of dissipation in the cochlear partition by means of a network representing the basilar membrane. $J$. Acoust. Soc. Am., 23:151-154, 1951.

[7] R. F. Chadwick. Studies in cochlear mechanics. In M. H. Holmes and A. Rubenfeld, editors, Mathematical modeling of the hearing process. Lecture Notes in Biomathematics, volume 43. Springer-Verlag, 1980.

[8] W. W. Clark, D. O. Kim, P. M. Zurek, and B. A. Bohne. Spontaneous otoacoustic emissions in chinchilla ear canals: correlation with histopathology and suppression by external tones. Hear. Res., 16:299-314, 1984.

[9] B. P. de Boer. Solving cochlear mechanics problems with higher order differential equations. J. Acoust. Soc. Am., 72:1427-1434, 1982.

[10] H. Fletcher. On the dynamics of the cochlea. J. Acoust. Soc. Am., 23:637-645, 1951.

[11] C. D. Geisler. From Sound to Synapse. Oxford University Press, New York, 1998.

[12] E. Givelberg. Modeling Elastic Shells Immersed in Fluid. PhD thesis, New York University, 1997.

[13] T. Gold. Hearing ii. the physical basis of the action of the cochlea. Proc $R$ Soc Lond [Biol], 135:492-498, 1948.

[14] M. H. Holmes. A mathematical model of the dynamics of the inner ear. $J$. Fluid Mech., 116:59-75, 1982.

[15] A. Inselberg and R. F. Chadwick. Mathematical model of the cochlea. i: formulation and solution. SIAM J. Appl. Math., 30:149-163, 1976.

[16] B. M. Johnstone and A. J. F. Boyle. Basilar membrane vibration examined with the mössbauer technique. Science, 158:390-391, 1967.

[17] D. T. Kemp. Stimulated acoustic emissions from within the human auditory system. J. Acoust. Soc. Am., 64:66-81, 1978.

[18] S. M. Khanna and D. G. B. Leonard. Basilar membrane tuning in the cat cochlea. Science, 215:305-306, 1982.

[19] D. O. Kim, C. E. Milnar, and R. R. Pfeiffer. A system of nonlinear differential equations modeling basilar membrane motion. J. Acoust. Soc. Am., 54:1517-1529, 1973.

[20] S. M. Kohllöffel. A study of basilar membrane vibrations ii. the vibratory amplitude and phase pattern along the basilar membrane (post-mortem). Acustica, 27:66-81, 1972.

[21] P. J. Kolston. Finite element micromechanical modeling of the cochlea in three dimensions. J. Acoust. Soc. Am., 99(1):455-467, 1996. 
[22] P. J. Kolston. Comparing in it vitro, in situ and in vivo experimental data in a three dimensional model of mammalian cochlear mechanics. Proc. Natl. Acad. Sci. USA, 96:3676-3681, March 1999.

[23] M. B. Lesser and D. A. Berkley. Fluid mechanics of the cochlea. part i. J. Fluid Mech., 51:497-512, 1972.

[24] R. J. Leveque, C. S. Peskin, and P. D. Lax. Solution of a two-dimensional cochlea model with fluid viscosity. SIAM J. Appl. Math., 48:191-213, 1988.

[25] C. H. Loh. Multiple scale analysis of the spirally coiled cochlea. J. Acoust. Soc. Am., 74:95-103, 1983.

[26] D. Manoussaki and R.S. Chadwick. Effects of geometry on fluid loading in a coiled cochlea. SIAM J. Appl. Math., 61:369-386, 2000.

[27] A. Nuttall and T. Ren. Extracochlear electrically evoked otoacoustic emissions: a model for in vivo assessment of outer hair cell electromotility. Hear. Res., 92:178-183, 1995.

[28] A. A. Parthasarati, K. Grosh, and A. L. Nuttall. Three-dimensional numerical modeling for global cochlear dynamics. J. Acoust. Soc. Am., 107(1):474-485, January 2000.

[29] C. S. Peskin. J. Comp. Phys., 25:20, 1977.

[30] C. S. Peskin. The immersed boundary method. Acta Numerica, 11:479-517, 2002.

[31] C. S. Peskin and D. M. McQueen. A general method for the computer simulation of biological systems interacting with fluids. In Proc. of SEB Symposium on Biological Fluid Dynamics, Leeds, England,, July 1994.

[32] C. S. Peskin and B. F. Printz. Improved volume conservation in the computation of flows with immersed elastic boundaries. J. Comp. Phys., 105:33-46, 1993.

[33] L. C. Peterson and B. P. Bogert. A dynamical theory of the cochlea. J. Acoust. Soc. Am., 22:369-381, 1950.

[34] S. Ramamoorthy, K. Grosh, and J. M. Dodson. Theoretical study of structural acoustic silencers for hydraulic systems. J. Acoust. Soc. Am., 111(5):2097-2108, 2002.

[35] O. F. Ranke. Theory of operation of the cochlea: A contribution to the hydrodynamics of the cochlea. J. Acoust. Soc. Am., 22:772-777, 1950.

[36] W. S. Rhode. Observations of the vibrations of the squirrel monkeys using the mössbauer technique. J. Acoust. Soc. Am., 49:1218-1231, 1971.

[37] L. Robles, M. Ruggero, and N. C. Rich. Mössbauer measurements of basilar membrane tuning curves in the chinchilla. J. Acoust. Soc. Am., 76(S35), 1984.

[38] M. R. Schroeder. J. Acoust. Soc. Am., 53:429-434, 1973. 
[39] P. M. Sellick, R. Patuzzi, and B. M. Johnston. Measurement of basilar membrane motion in the guinea pig using the mössbauer technique. J. Acoust. Soc. Am., 72:131-141, 1982.

[40] W. M. Siebert. Ranke revisited - a simple short-wave cochlear model. J. Acoust. Soc. Am., 56:596-600, 1974.

[41] C. R. Steele. Behaviour of the basilar membrane with pure-tone excitation. J. Acoust. Soc. Am., 55:148-162, 1974.

[42] C. R. Steele and L. A. Taber. Comparison of wkb calculations and experimental results for three-dimensional cochlear models. J. Acoust. Soc. Am., 65:1007-1018, 1979.

[43] C. R. Steele and J. G. Zais. Effect of coiling in a cochlear model. J. Acoust. Soc. Am., 77(5):1849-1852, 1985.

[44] M. A. Viergever. Basilar membrane motion in a spiral shaped cochlea. J. Acoust. Soc. Am., 64:1048-1053, 1978.

[45] G. von Békésy. Experiments in Hearing. Mc-Graw Hill, New York, 1960.

[46] G. Zweig, R. Lipes, and J. R. Pierce. The cochlear compromise. J. Acoust. Soc. Am., 59:975-982, 1976.

[47] J. J. Zwislocki. Analysis of some auditory characteristics. In R. R. Buck R. D. Luce and E. Galanter, editors, Handbook of Mathematical Psychology. Wiley, New York., 1965.

[48] J. J. Zwislocki and J. Moscicki. Theorie der schenkenmechanik - qualitative und quantitative ananlyse. Acta Otolaryng.(suppl.), 72, 1948. 